\title{
Comparison of Disc Diffusion, Broth Microdilution and Modified Hodge Test Susceptibility Testing of Escherichia coli Isolates to Beta-Lactam Antibiotics
}

\author{
Hesam Alizade (PhD) \\ Research Center for Tropical and \\ Infectious Diseases, Kerman \\ University of Medical Sciences, \\ Kerman, Iran \\ Fatemeh Fallah (PhD) \\ Department of Microbiology, Faculty \\ of Medicine, Shahid Beheshti \\ University of Medical Science, Tehran, \\ Iran \\ Reza Ghanbarpour (PhD) \\ Department of Microbiology, Faculty \\ of Veterinary Medicine, Shahid \\ Bahonar University, Kerman, Iran \\ Hosein Goudarzi (PhD) \\ Department of Microbiology, Faculty \\ of Medicine, Shahid Beheshti \\ University of Medical Science, Tehran, \\ Iran \\ Hamid Sharifi (PhD) \\ Modeling in Health Research Center, \\ Institute of Future Studies in Health, \\ Kerman University of Medical \\ Sciences, Kerman, Iran \\ Mohammad Reza Aflatoonian \\ (DVM, MPH) \\ Research Center for Tropical and \\ Infectious Diseases, Kerman \\ University of Medical Sciences, \\ Kerman, Iran \\ Corresponding Author: Mohammad \\ Reza Aflatoonian \\ Email: alizade.h2000@yahoo.com \\ Tel: +989133982084 \\ Adderess: Research Center for \\ Tropical and Infectious Diseases, \\ Kerman University of Medical \\ Sciences, Kerman, Iran
}

Received : 04 jan 2015

Revised: 19 jan 2015

Accepted: 24 Feb 2015

\section{ABSTRACT}

Background and Objective: 0ne of the main tasks of clinical microbiology laboratories is to determine antibiotic resistance profiles in common pathogens and ensure the selection of effective antibiotics for certain infections. The aim of this study was to compare the methods of disk diffusion, broth microdilution and modified Hodge test in Escherichia coli isolates from urinary tract infection and diarrhea for susceptibility testing against beta-lactam antibiotics in Kerman, Iran.

Methods: In this study, 432 E. coli isolates were collected from diarrhea (216 isolates) and urinary tract infection samples (216 isolates). The antibiotic susceptibility testing methods of disk diffusion, broth microdilution and modified Hodge test were performed according to the Clinical and Laboratory Standards Institute guidelines.

Results: The findings of disk diffusion method showed that resistance to cefotaxime, ceftazidime, aztreonam, cefepime and imipenem was 51.15\%, 30.55\%, 24.30\%, 15.27\% and $1.85 \%$, respectively. In the disk diffusion test, $51.15 \%$ of the isolates were resistant to at least one antibiotic, all of which were later evaluated by the broth microdilution method. Moreover, $52.94 \%, 17.19 \%, 13.12 \%$ and $0.90 \%$ of the isolates were resistant to cefotaxime, ceftazidime, cefepime and imipenem, respectively. All of the isolates were evaluated for the production of carbapenemase enzyme by the modified Hodge test and none of the isolates were found as positive.

Conclusion: This study shows that performing carbapenem tests is very challenging, and laboratories are recommended to use secondary and independent antibiotic susceptibility tests such as modified Hodge test to confirm the carbapenem-resistant results.

Keywords: Escherichia coli, Disk Diffusion Antimicrobial Tests, Beta-Lactams.

This paper should be cited as: Alizade H, Fallah F, Ghanbarpour R, Goudarzi H, Sharifi H, Aflatoonian HR. [Comparison of Disc Diffusion, Broth Microdilution and Modified Hodge Test Susceptibility Testing Of Escherichia coli Isolates to Beta-Lactam Antibiotics]. mljgoums. 2016; 10(2):19 -24 


\section{INTRODUCTION}

Performance of antimicrobial susceptibility tests by clinical microbiology laboratories is important to confirm the sensitivity to antimicrobial agents or detect antibiotic resistance in certain bacterial isolates. These antimicrobial susceptibility tests are also important for bacteria such as members of the family Enterobacteriaceae, Pseudomonas species, Staphylococcus species and Enterococcus species, which may acquire important antibiotic resistance mechanisms (1). Pathogenic strains of E. coli are considered as one of the most important causes of gastrointestinal and non-gastrointestinal infections. It also causes a wide range of extraintestinal infections such as urinary tract infection (UTI), septicemia, neonatal meningitis and infections of the central nervous system that leads to mortality and increased cost of treatment (2,3). Among the clinical isolates that produce extendedspectrum $\beta$-lactamases (ESBL) enzymes, the members of the family Enterobacteriaceae, particularly $E$. coli show resistance against $\beta$ lactam antibiotics. These antibiotics are normally used to treat several nosocomial and community-acquired infections (4).

Carbapenems are effective as the last line of antibiotics defence against fermentative and non-fermentative bacteria involved in majority of common nosocomial infections such as UTI, respiratory infections, wound infections etc. Hodge modified test is a method with a high sensitivity and specificity (>90\%) for detection of carbapenemase-producing isolates $(5,6)$. The correct diagnosis of clinical microbiology laboratories is necessary to identify ESBL enzymes using combination disc test, which is often performed by the routine method of disk diffusion. In addition, the Clinical and Laboratory Standards Institute (CLSI) stated the disk diffusion and broth microdilution methods as the standard tests to confirm the identification of E. coli, Klebsiella pneumonia and ESBL enzyme-producing $K$. oxytoca (6). The broth microdilution method can be performed using panels and commercial reagents due to its repeatability. Computer reports can also be presented through reading the automatic panel. However, the main disadvantage of broth microdilution method is the difficulty in the lack of choosing some antibiotics in the commercial panels. The lack of mechanization or automation is a disadvantage of the disk diffusion method (1). It is of great importance to identify $\beta$-lactamresistant bacteria correctly as well as the bacteria capable of producing ESBL- and metallo-beta-lactamase (MBL) enzymes that play a fundamental role in the development of resistance to these antibiotics. Thus, this study aimed to compare the disc diffusion, Hodge modified test and broth microdilution method for identification of isolates that produce ESBL and MBL enzymes, in addition to evaluating these methods in relation to the effect of $\beta$-lactam antibiotics on $E$. coli isolates from clinical samples.

\section{MATERIAL AND METHODS}

In this study, 432 E. coli isolates were collected from UTI samples (216 isolates) and diarrhea samples (216 isolates) obtained from the laboratories in the city of Kerman, during a period of 8 months in 2013. The isolates were identified on specific culture media using standard bacteriological and biochemical tests. The approved isolates were kept in LuriaBertani broth (Biolife Laboratory, Milan, Italy) with $30 \%$ glycerol at $-70{ }^{\circ} \mathrm{C}$ until the start of experiments and determination of antibiotic susceptibility.

Broth microdilution method

The Minimum Inhibitory Concentration (MIC) was determined using the broth microdilution method according to the CLSI protocol (2013) (6). Powders of ceftazidime $(1 \mu \mathrm{g} / \mathrm{mL})$, cefotaxime $(1 \mu \mathrm{g} / \mathrm{mL})$, imipenem $(1 \mu \mathrm{g} / \mathrm{mL})$, cefepime $(1 \mu \mathrm{g} / \mathrm{mL})$ and meropenem (1 $\mu \mathrm{g} / \mathrm{mL}$ ) were added. After 24 hours of incubation at $37{ }^{\circ} \mathrm{C}$, the turbidity was assessed to indicate bacterial growth and results were later recorded in a specific table. According to the definition, the concentration (most diluted) of the last well with no turbidity was determined as the MIC. The antibiotic powders used in this study were purchased from Glasgow Co. (UK) and HiMedia Co. (India).

The Kirby and Bauer method was used for determination of antibiotic susceptibility of the isolates according to the guidelines of CLSI (2013) (6). The Mueller-Hinton agar medium (Merck, Germany) was used to perform disk diffusion method. Cefepime $(10 \mu \mathrm{g})$, ceftazidime $(30 \mu \mathrm{g})$, cefotaxime $(30 \mu \mathrm{g})$, 
imipenem $(10 \mu \mathrm{g})$ and aztreonam $(30 \mu \mathrm{g})$ disks were purchased from the Padtan Teb Co. (Iran) for this study.

test was performed to detect carbapenemaseproducing isolates according to the guidelines of CLSI (2013) (6). Thus, a suspension of the reference strain of E. coli ATCC 25922 was cultured on Mueller-Hinton agar equal to a 0.5 McFarland standard. This strain is susceptible to the carbapenem antibiotics (imipenem and ertapenem). Ertapenem disk (Padtan Teb, Iran) was placed in the center of the plate and streak culture of the isolates and controls was done from the edge of the disk to the end of the plate. The plates were incubated overnight. If the isolates tested are ertapenem-susceptible, a complete halo appears around the disc. However, if the isolates are resistant to ertapenem, the created zone will be incomplete (in the form of cloverleaf) and growth of the reference strain of E. coli ATCC 25922 becomes apparent around the isolates.

\section{RESULTS}

The results of antimicrobial susceptibility tests by the disk diffusion method showed that 221 isolates $(51.15 \%)$ were cefotaxime-resistant, 132 (30.55\%) were ceftazidime-resistant, $105 \quad(24.30 \%)$ were aztreonam-resistant, $66 \quad(15.27 \%)$ were cefepime-resistant and $8(1.85 \%)$ were resistant to imipenem. The highest and lowest levels of resistance in the disk diffusion method for UTI and diarrhea isolates were observed against cefotaxime and imipenem, respectively (Table 1).

Broth microdilution test was performed on 221 isolates from UTI and diarrhea samples that were resistant against at least one antibiotic in the disk diffusion method. The highest rate of resistance was seen against cefotaxime (117 isolates), ceftazidime (96 isolates), cefepime (63 isolates) and imipenem (2 isolates), respectively. None of the isolates in the broth microdilution method were meropenemresistant. The highest level of resistant among the UTI isolates was against ceftazidime, while none of the isolates were resistant against imipenem and meropenem. Diarrheal isolates showed the highest rate of resistance against cefotaxime and none of them was resistant against meropenem (Table 1).

None of the isolates was reported as positive based on the results obtained from the isolates of UTI and diarrhea in the modified Hodge test to confirm the MBL enzymes.

Table 1- Antibiotic resistance among the $E$. coli isolates from UTI and diarrhea samples in the disk diffusion and broth microdilution methods

\begin{tabular}{ccccc} 
& \multicolumn{2}{c}{ disk diffusion } & \multicolumn{2}{c}{ broth microdilution } \\
\cline { 2 - 5 } & Diarrhea $(\%)$ & UTI* $(\%)$ & Diarrhea $(\%)$ & UTI $(\%)$ \\
Cefotaxime & $\mathbf{8 8}(40.74)$ & $133(61.57)$ & $61(69.31)$ & $\mathbf{5 6}(42.10)$ \\
Ceftazidime & $\mathbf{5 5}(25.46)$ & $77(35.64)$ & $\mathbf{3 8}(43.18)$ & $\mathbf{8 2}(\mathbf{6 1 . 6 5})$ \\
Cefepime & $\mathbf{3 0}(13.88)$ & $\mathbf{3 6}(\mathbf{1 6 . 6 6 )}$ & $\mathbf{2 9}(32.95)$ & $\mathbf{3 4}(\mathbf{2 5 . 5 4 )}$ \\
Aztreonam & $\mathbf{4 8}(22.22)$ & $\mathbf{5 7}(\mathbf{2 6 . 3 8})$ & - & - \\
Imipenem & $\mathbf{6}(2.77)$ & $\mathbf{2 ( 0 . 9 2 )}$ & $\mathbf{2}(\mathbf{2 . 2 7})$ & $\mathbf{0 . 0 0}$ \\
Meropenem & - & - & $\mathbf{0 . 0 0}$ & $\mathbf{0 . 0 0}$ \\
\hline
\end{tabular}




\section{DISCUSSION}

In this study, the antibiotic susceptibility of the isolates to five antibiotics was assessed. In the disk diffusion method, the highest level of resistance was to cefotaxime and ceftazidime, while the lowest level of resistance was to cefepime and imipenem. The resistant strains in the disk diffusion method were evaluated by broth microdilution method and the highest level of resistance was related to cefotaxime and ceftazidime, while the lowest level to meropenem and imipenem. However, none of the isolates were positive for production of carbapenemase enzyme in the modified Hodge test.

Based on the results of this study, two E. coli isolates from the diarrhea samples were imipenem-resistant in the broth microdilution method, although none of the isolates were positive for production of carbapenemase enzyme in the modified Hodge test. However, the modified Hodge test is considered a weakly positive test for carbapenemaseproducing $E$. coli isolates and a strong positive test for the carbapenemase-producing $K$. pneumoniae isolates (7).

Both disc diffusion and broth microdilution methods have their own advantages and disadvantages that are discussed below. An advantage of the broth microdilution method is that it can express the results quantitatively, while the main disadvantages of this method is the state of boredom and exhaustion during the experiment. Manual preparation of antibiotic solutions for each test, the possibility of introducing errors in the antibiotic solution and relatively high amount of space and reagents required for each test are other disadvantages of the broth microdilution method. The Disk diffusion is a simple method that does not require special tools, enables physicians to interpret definite results easily and the disks can be chosen conveniently for testing. This test is the most cost-effective method of antibiotic susceptibility testing (about 2-5 dollars per test for materials used) and the qualitative results are expressed in three forms of susceptible, intermediate or resistant. The disadvantage of this method is that all difficult to grow and slow-growing bacteria cannot be accurately assessed in this method. For bacteria such as Streptococcus spp, Haemophilus influenzae and Neisseria meningitidis, this method should be performed using standard procedures, specific culture medium, incubation conditions and certain terms for interpretation of the size of the growth inhibition zone $(8,9)$.

The function of tools for antibiotic susceptibility testing should be constantly monitored and updated if required, due to the emergence of new antimicrobial resistance mechanisms, including resistances that are difficult to identify (such as some carbapenemase-producing Gram-negative bacteria and antibiotic susceptibility testing of Staphylococcus aureus to vancomycin). In some cases, it is necessary to use specific methods such as the modified Hodge test for carbapenemase-producing bacteria to complete routine tests (1). Torres et al. study (2009) compared disk diffusion method, E-test and VITEK 2 system on susceptibility testing of $P$. aeruginosa to $\beta$-lactam antibiotics (piperacillin, piperacillin-tazobactam, ceftazidime, cefepime, tazobactam and imipenem). The results of the mentioned study were compared with the broth microdilution method, which is proposed by the CLSI as the standard method. Very large errors that are showed as false sensitive were only identified by the disk diffusion method for tazobactam and cefepime, while all three methods could identify imipenem. The major errors that are reported as false resistant were generally acceptable for all antibiotics except tazobactam. VITEK 2 system showed a high level of minor errors (a tendency toward false sensitivity) particularly to ceftazidime and cefepime. In general, E-test showed a high rate of false-resistant results to all antibiotics among the tested methods, while the disk diffusion method showed a high rate of falsesusceptible results to all antibiotics except ceftazidime (10).

broth microdilution method, which showed that only 11 isolates were moderately resistant or resistant to imipenem and meropenem. Among the Enterobacteriaceae isolates, 13 E. moderately susceptible and resistant isolates in the mentioned study were re-evaluated by the resistant to imipenem and meropenem. The coli isolates were reported as imipenem and family were moderately susceptible and meropenem- susceptible or moderately areas, 
123 isolates of the Enterobacteriaceae susceptible by the laboratories, while this was not approved in any of the isolates in the broth microdilution method (11).

In line with this study, a study in 2003 evaluated the antibiotic susceptibility tests including agar dilution, disk diffusion, E-test and VITEK 2 on the above isolates. The results of each test were then compared with the broth microdilution method. In the broth microdilution method, seven isolates were resistant to imipenem and five meropenemresistant isolates were identified from the Enterobacteriaceae family.

The methods investigated in this study showed few large and very large errors. These studies did not have a satisfactory explanation for the large number of isolates that were reported as false-moderately sensitive or resistant to imipenem, but demonstrated that there are problems for testing carbapenem. Therefore, laboratories should use independent alternative antibiotic susceptibility tests such as the modified Hodge test, to confirm results that are moderately sensitive and resistant to carbapenem (12). In addition to carbapenemase enzymes, the emergence of bacterial resistance to carbapenem antibiotics and false-resistance or false-sensitive results in carbapenemase diagnostic test may be due to other mechanisms such as changes in the permeability of the outer membrane, ESBL enzymes, over-expression of AmpC and purine-loss that can be accompanied by cephalosporinase (6).

\section{CONCLUSION}

Antibiotic susceptibility testing through the disk diffusion method shows that most isolates

\section{REFERENCES}

1. Jorgensen JH, Ferraro MJ. Antimicrobial Susceptibility Testing: A Review of General Principles and Contemporary Practices. Clin Infect Dis. 2009; 49(11): 1749-55. doi: 10.1086/647952.

2. Alizade H, Ghanbarpour R, Aflatoonian, MR. Virulence genotyping of Escherichia coli isolates from diarrheic and urinary tract infections in relation to phylogeny in southeast of Iran. Trop Biomed. 2014; 31(1): 174-82. PMID:24862058.

3. Hemati Z, Ghanbarpour R, Alizade H. The Distribution of beta-lactamase genes in Escherichia coli phylotypes isolated from diarrhea and UTI Cases in Northwest Iran. Adv Clin Exp Med. 2014; 23(4): 523-9. PMID:25166436. from UTIs are more resistant to cefotaxime, ceftazidime, aztreonam and imipenem in comparison with the isolates from diarrhea. The laboratory experts should correctly perform antibiotic susceptibility tests. They should also identify and inform the physicians about the mechanisms of drug resistance, particularly the most important forms such as beta-lactamases resistance. Moreover, there are some difficulties involved in carbapenem resistance testing. Thus, laboratories are recommended to use independent alternative antibiotic susceptibility tests such as the modified Hodge test to confirm results that are moderately sensitive and resistant to carbapenem. Porin-loss, AmpC beta-lactamase enzymes or/and CTX-M are among the reasons for false-resistant and false-sensitive results in the modified Hodge test. However, the tests including PCR, western blotting and finally sequencing can resolve these results. The exact cause of resistance of a bacterium to various antibiotics should be identified in the laboratory to determine the best treatment option for patients.

\section{ACKNOWLEDGEMENT}

This study was part of a research project sponsored by the Research Center for Tropical and Infectious Diseases, Kerman University of Medical Sciences, Kerman, Iran (Number: IT/92/106).

\section{CONFLICT OF INTEREST}

The author(s) declared no potential conflicts of interest with respect to the research, authorship, and/or publication of this article.

4. Wollheim C, Guerra IMF, Conte VD, Hoffman SP, Schreiner FJ, Delamare APL, et al. Nosocomial and community infections due to class A extended-spectrum $\beta$-lactamase producing Escherichia coli and Klebsiella spp. in southern Brazil. Braz J Infect Dis. 2011; 15(2): 138-43. PMID:21503400.

5. Fallah F, Taherpour A, Hakemi Vala M, Hashemi A. Global spread of New Delhi metallo-beta-lactamase-1 (NDM-1). Iran J Clin Infect Dis. 2011; 6(4): 171-7.

6. Clinical and Laboratory Standards Institute. Performance standards for antimicrobial susceptibility testing; Twenty-third informational supplement. M100S23 Wayne, PA: CLSI; 2013; 33(1). 
7. Birgy A, Doit C, Mariani-Kurkdjian P, Genel N, Faye A, Arlet G, et al. Early detection of colonization by VIM1-producing Klebsiella pneumoniae and NDM-1producing Escherichia coli in two children returning to France. J Clin Microbiol. 2011; 49(8): 3085-7. doi: 10.1128/JCM.00540-11.

8. Jorgensen JH, Turnidge JD. Antibacterial susceptibility tests: dilution and disk diffusion methods. Manual of clinical microbiology. 9th ed. Washington, DC: American Society for Microbiology. 2007; 1152-72.

9. Clinical and Laboratory Standards Institute. Methods for dilution antimicrobial susceptibility testing for bacteria that grew aerobically. Approved Standard M7A10. Wayne, PA. 2009.

10. Torres E, Villanueva R, Bou G. Comparison of different methods of determining $\beta$-lactam susceptibility in clinical strains of Pseudomonas aeruginosa. J Med Microbiol. 2009; 58(Pt 5): 625-9. doi: 10.1099/jmm.0.005587-0.

11. Steward CD, Wallace D, Hubert SK, Lawton R, Fridkin SK, Gaynes RP, et al. Ability of laboratories to detect emerging antimicrobial resistance in nosocomial pathogens: a survey of project ICARE laboratories. Diagn Microbiol Infect Dis. 2000; 38(1): 59-67. PMID:11025185.

12. Steward CD, Mohammed JM, Swenson JM, Stocker SA, Williams PP, Gaynes RP, et al. Antimicrobial susceptibility testing of carbapenems: multicenter validity testing and accuracy levels of five antimicrobial test methods for detecting resistance in Enterobacteriaceae and Pseudomonas aeruginosa isolates. J Clin Microbiol. 2003; 41(1): 351-8. 